\title{
An Adjustable Sensor Platform Using Dual Wavelength Measurements For Optical Colorimetric Sensitive Films
}

\author{
Carlos Machado ${ }^{*}$, Carlos Gouveia ${ }^{+}$, João Ferreira ${ }^{+}$, Barna Kovacs ${ }^{\#}$, Pedro Jorge ${ }^{+}$, Luis Lopes ${ }^{*}$ \\ "Department of Computer Science, Faculty of Science, University of Porto, Porto, Portugal, \\ \{carlos.machado,luis.lopes\}@dcc.fc.up.pt \\ ${ }^{+}$INESC Porto, Rua do Campo Alegre, 687, 4169007 Porto, Portugal \\ "University of Pécs, Department of General and Physical Chemistry, Pécs, Hungary
}

\begin{abstract}
We present a new and versatile sensor platform to readout the response of sensitive colorimetric films. The platform is fully self-contained and based on a switched dual-wavelength scheme. After filtering and signal processing, the system is able to provide self-referenced measures of color intensity changes in the film, while being immune to noise sources such as ambient light and fluctuations in the power source and in the optical path. By controlling the power and the switching frequency between the two wavelengths it is possible to fine tune the output gain as well as the operational range of the sensor for a particular application, thus improving the signal conditioning. The platform uses a micro-controller that complements the analog circuit used to acquire the signal. The latter pre-amplifies, filters and conditions the signal, leaving the micro-controller free to perform sensor linearization and unit conversion. By changing the sensitive film and the wavelength of the light source it is possible to use this platform for a wide range of sensing applications.
\end{abstract}

Keywords- Optical Sensors; Self-Contained; Colorimetric Sensitive Film; MCU; Filters; Self-Referenced; PWM; Adjustable Sensor; Dual Wavelength Measurements; Colorimetry; Thin Films

\section{INTRODUCTION}

The measurement and control of chemical and biological parameters in environmental, industrial and biomedical applications is of great importance [1]. In this context, sensitive films that change their optical properties when in contact with specific chemical compounds provide a promising technology for the development of effective, low cost, colorimetric sensors for a wide range of applications. For example, one of the most popular biochemical optical sensing mechanisms relies on the changes of the colorimetric (absorption) properties of an indicator dye [2]. In this case, the intensity of the light passing through the film is a function of the concentration of the chemical compound being investigated. Low-cost, maturity, high sensitivity and availability of a wide range of materials, are some of the advantages associated with this technique. In addition, performance parameters such as sensitivity and response time can be controlled through the film thickness. These characteristics motivated the design and implementation of a generic, self-contained, sensor platform that allows the measurement of the concentration of chemical compounds simply by adjusting software parameters. A pulse width modulation (PWM) approach enables self-referenced dual wavelength detection using a single photodetector.

The platform's low power consumption and low cost should also make it attractive for use in wireless sensor networks or high autonomy portable chemical sensor devices.

\section{EXPERIMENT}

A wide range of colorimetric sensitive films are available to measure different chemical analytes such as: carbon dioxide $\left(\mathrm{CO}_{2}\right), \mathrm{pH}$, ammonia $\left(\mathrm{NH}_{3}\right)$, and others [3]. In the experiment presented in this paper, we used a $\mathrm{CO}_{2}$ sensitive layer that changes its absorbance as a function of the concentration of the gas.

The $\mathrm{CO}_{2}$ sensitive layer is based on the acid-basic equilibrium of phenol and of its derivative 4-nitro-phenol. They are kept in their deprotonated form in the sensing membrane. In the presence of carbon dioxide, hydrogen carbonate is formed that partially protonates the phenols. The hydroxyl group is involved in protolytic reactions that modify the charge distribution in the molecule. As result of the interaction with carbon dioxide, a change in absorption is observed due to the delocalization of the electrons in the aromatic ring of these compounds. A quaternary ammonium compound, didodecyl-dimethyl-ammonium hydroxide (DDMA) was used to deprotonate the phenols, preparing them for being protonated by the analyte, also providing the reversibility of the response. The absorption band is observed around the wavelength of $430 \mathrm{~nm}$. For more details about this work, please see [4].

\section{A. Method}

The sensing polymer layer was prepared from a $5 \%(\mathrm{~m} / \mathrm{m})$ polyurethane hydrogel (D4) dissolved in ethanol solution. Then $50 \mu \mathrm{l}$ of $0.1 \mathrm{M}$ ethanolic 4-nitro-phenol and $100 \mu \mathrm{l}$ of $0.1 \mathrm{M}$ ethanolic DDMA solution were added to $500 \mu \mathrm{l}$ of polymer solution. The resulting solution was spread on Mylar foil by spin coating and allowed to dry for 4 hours. To carry out the measurement, the sensitive film was coupled to the developed system with appropriate excitation and detection optoelectronics. 
As stated before, a dual-wavelength LED is used (LED430/565-04A, from Roithner Lasertechnik GmbH). One wavelength $(430 \mathrm{~nm})$ coinciding with the sensitive film absorption band, herein called Sensitive Light (SL), whose measured intensity will change as it is absorbed by the film, as a function of the concentration of gas. The second wavelength $(565 \mathrm{~nm})$, called Reference Light (RL), is used as a reference signal. The measurement at this wavelength is not affected by the absorption change of the film, and is used to compensate eventual optical power fluctuations on the measurements due to optical path losses, misalignments and power supply circuit.

A photodiode was used as a detector for both the SL and the RL, as they travel through the sensitive film. The distance between the light source and the photodiode can be made very small as the measurement is based on the different responses of the SL and RL to the film, as opposed to [5, 6] NDIR sensors.

The dual-wavelength source can be used in parallel, but that would require more complex emitting and receiving circuits in order to modulate and filter the two light signals. Thus, in order to simplify the circuit, we used a timemultiplexing scheme that repeatedly switches between the SL and RL wavelengths.

The control of the power and of the switching frequency for the light source was implemented using a simple, low power, micro-controller, providing the extra flexibility of changing these parameters directly by software.

\section{B. Architecture}

The overall architecture of the sensor platform is depicted in Figure 1. The micro-controller unit (MCU) is the core that controls the hardware functions and the operation of the platform sensor. In order to be able to interact with the physical world the platform requires power and signal circuit electronics. The power electronics circuit is responsible for controlling the power supply and the power required by the sensor platform. The signal circuit electronics handles signal amplification and analog signal processing.

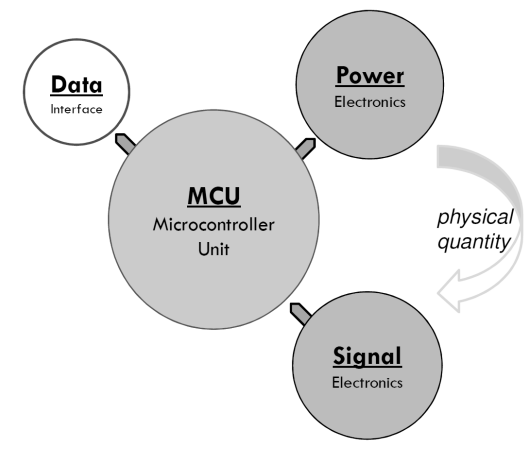

Fig. 1. MCU sensor, with data, signal and power electronics components.

The MCU captures the analog signal, performs some signal clean up and sensor linearization. Finally the MCU is also responsible for defining the interface with the outside world by implementing a communication protocol that offers the measured data, converted from the captured raw data, in the correct units.

\section{Prototype}

We have implemented a hardware prototype of the architecture presented in the previous section (Figure 2). The goal is to develop a low cost, low power, versatile and standalone platform to probe colorimetric sensitive films for a wide range of applications.

The prototype was built as an Arduino Compatible Shield to allow for rapid prototyping and integration with other types of sensors or communication interfaces, as in wireless sensor networks. Nevertheless, it can be used as a stand-alone unit using just a USB connection for data acquisition and for control. In this mode, the platform uses the USB connection as data and power supply sources.

In order to test the platform adjustment capability and functions we used a testing film scale. This scale consists of a strip of transparent acetate dyed with color gradient that mimics the $\mathrm{CO}_{2}$ sensitive film, i.e., the film scale transmitted less and less light in the $430 \mathrm{~nm}$ wavelength of the SL, mimicking an increasing $\mathrm{CO}_{2}$ ambient concentration.

The photodiode will detect a decreasingly flux from the SL. At the same time, the flux from the RL source, at $565 \mathrm{~nm}$, is not affected, the photodiode detecting essentially a constant flux.

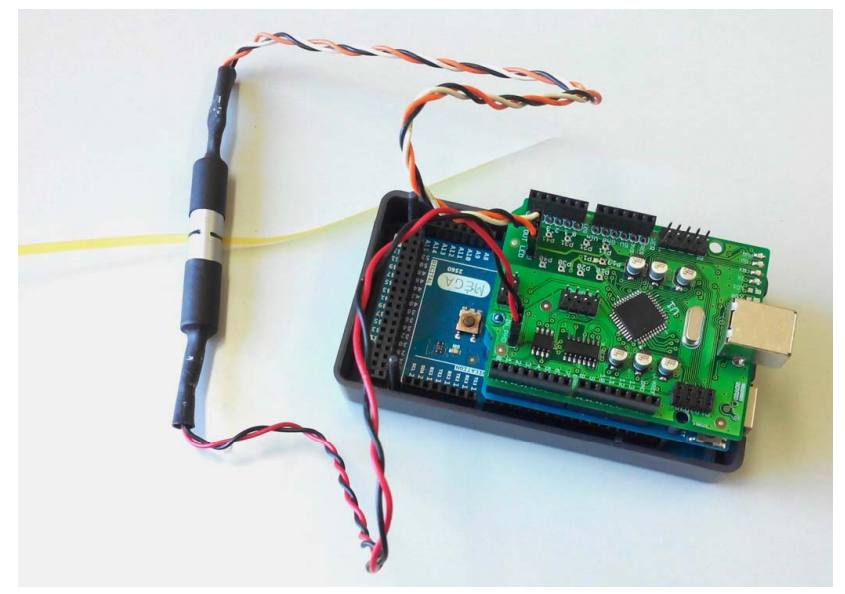

Fig. 2. Sensor board shield mounted on Arduino and color testing film scale.

\section{1) Sensor Platform signal processing path}

The signal processing path is illustrated in Figure 3. The measurement of the gas concentration starts with the MCU controlling the power of the dual-wavelength LED and the switching frequency between the SL and the RL, using the power electronics platform component.

The platform's analog signal electronics component is responsible for amplifying, filtering, processing and accommodating the signal captured from the photodiode.

Finally, this processed signal is captured by the MCU analog-to-digital converter (ADC), which calibrates the signal and converts it to the correct units. The final value can be accessed directly via USB interface to the platform shield or via the Arduino pins using the RS232 TTL interface. 


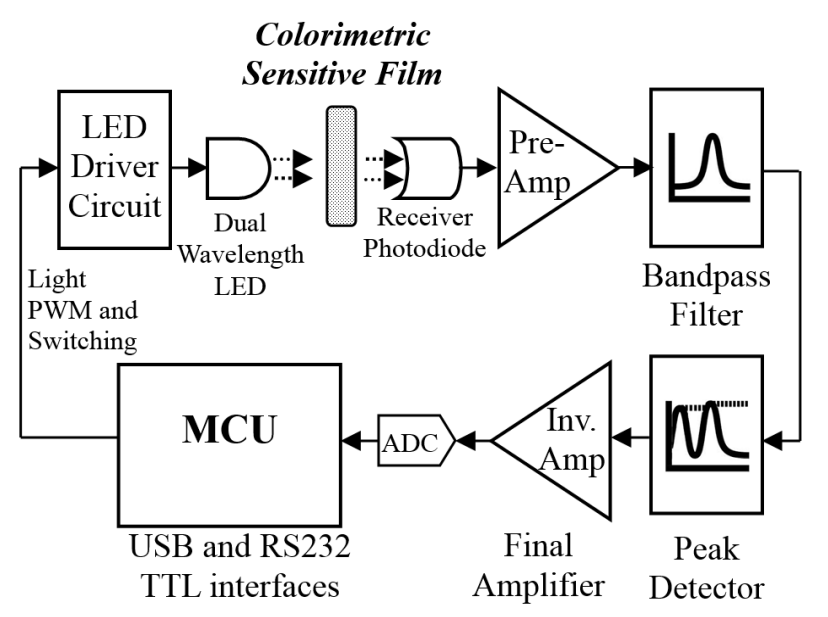

Fig. 3. Block schematic of sensor platform signal processing path.

\section{2) Power electronics circuit component}

The power electronics circuit is a transistor-based driver that regulates the energy supply of the two LEDs, individually. The power of the LEDs is easily controlled by the MCU using PWM at $80 \mathrm{kHz}$ to facilitate the later filtering. Only one light source is activated at given time. Switching of the light wavelengths is done with a frequency around $2.5 \mathrm{kHz}$ (configurable). Figure 4 shows the signals that drive the two LEDs.

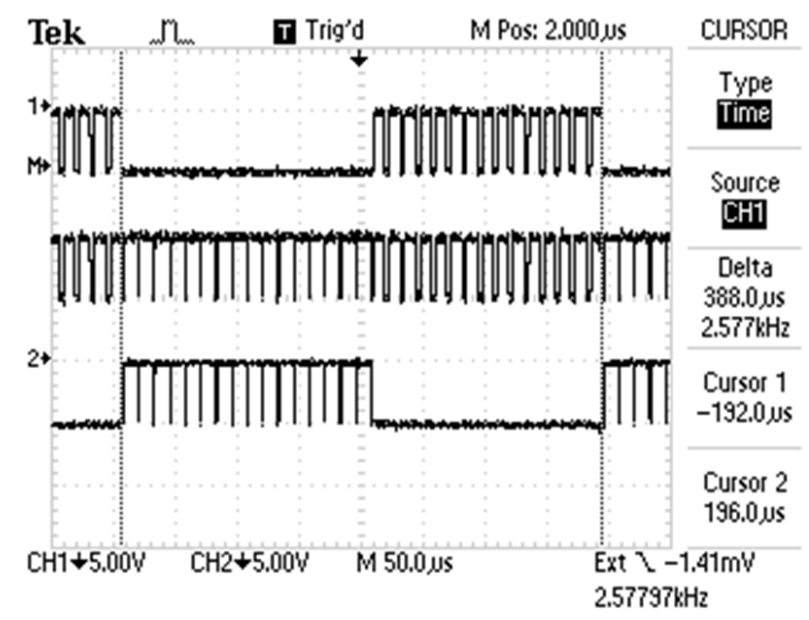

Fig. 4. Light PWM and switching between the two wavelengths. $(\{1 \rightarrow\}$ Upper trace - Reference light; $\{\mathrm{M} \rightarrow\}$ Middle trace - Sum of the two wavelengths; $\{2 \rightarrow\}$ Lower trace - Sensitive light).

The power electronic circuit is where the power is mainly consumed, having a typical consumption around $100 \mathrm{~mW}$ when the LEDs are activated, but this consumption only happens when the sensor is activated. The power circuit can be placed in a disabled mode, having no power consumption.

3) Signal electronics circuit component

The signal electronics circuit component uses operational amplifiers (OpAmp) to amplify, filter, process and conduct the signal.

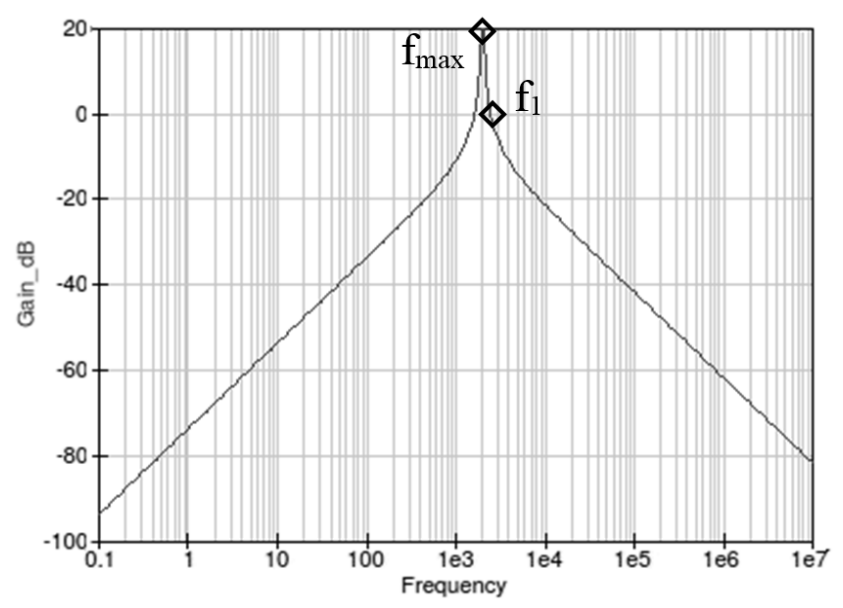

Fig. 5. $2^{\text {nd }}$ order active bandpass filter frequency response. By adjusting the switching light frequency the desired amplification can be achieved; $\left(\mathrm{f}_{\max }\right.$ - maximum gain frequency $(20 \mathrm{~dB}) ; \mathrm{f}_{1}$ - unitary gain frequency $\left.(0 \mathrm{~dB})\right)$.

The first stage is a transimpedance amplifier responsible to amplify the signal current from the photodiode and convert it to a voltage. The amplification is fixed with $\mathrm{x} 100$ gain $(40 \mathrm{~dB})$ that is sufficient without causing saturation of the next stage.

After the initial amplification, the signal passes through an active $2^{\text {nd }}$ order band pass filter. This filter is responsible to remove the PWM signal artefacts, as well as, removing eventual ambient light interference, like the $100 \mathrm{~Hz}$ introduced by lighting. The central frequency of the band filter is fixed around $2.5 \mathrm{kHz}$, defined by the switching frequency between the two wavelengths. Taking advantage of filter frequency response, shown in Fig 5, a shift in the switching frequency from the central frequency makes it possible to achieve different amplifications at the output. With this shift an adjustable output gain is achieved with a static circuit, as the switching light frequency is produced by the MCU.

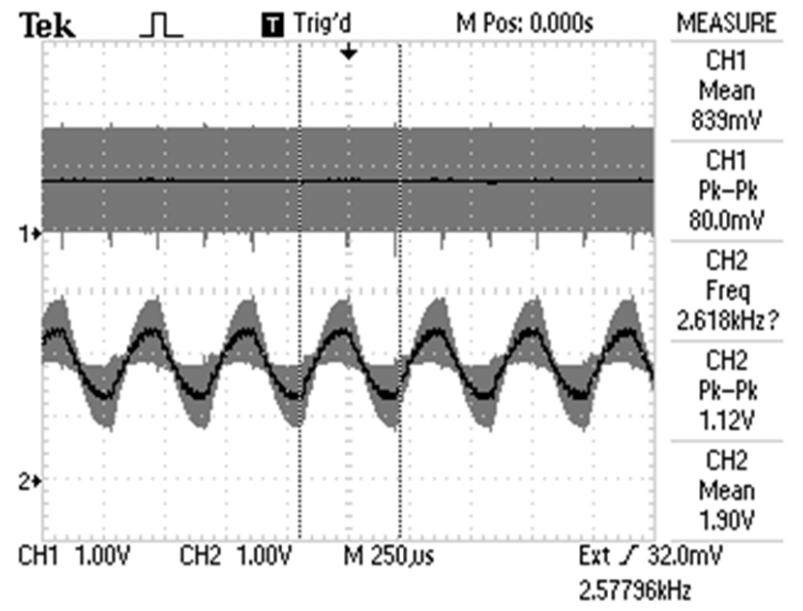

Fig. 6. Intermediate Analog circuit signals: $\{1 \rightarrow\}$ Upper Trace - Output signal before ADC; $\{2 \rightarrow\}$ Lower Trace - Signal after bandpass filter. The output working range is represented in gray. 
The resulting signal after the active band filter setup is the sinusoid wave, shown on Fig 6, with the frequency equal to the switching light frequency and where the amplitude is proportional to the colorimetric sensitive film light absorbance.

The amplitude information is captured using a peak detector implemented with OpAmp precision rectifier. Finally the signal is inverted and amplified in order to be captured by the ADC of the MCU, as shown on Fig 6.

All the hardware is supplied with a $5 \mathrm{~V}$ single voltage, which enforce the usage of supply filtering for the components of the platform and require the usage of low power rail-to-rail OpAmp. To be able to reduce the power consumption, the analog circuit power is controlled by a MOSFET that shutdowns the supply of the circuit on sensor disable state.

\section{4) Microcontroller Unit and Interface}

The sensor platform uses the ATMEGA16U4 micro-controller that is capable to generate the PWM outputs, it includes 10bit ADCs and features a USB 2.0 or TTL serial USART interfaces. All these peripherals are controlled by the MCU that is capable of six levels of low-power sleep modes. To interface with the platform, a Hayes Command Set is used, at $9600 \mathrm{bps}$, when using the TTL serial USART.

\section{RESULTS}

\section{A. Experiment Results}

The system was tested by using a $\mathrm{CO}_{2}$ sensitive film as indicated earlier. The sensing platform was subjected to steep variation of $\mathrm{CO}_{2}$ from $2 \%$ to $0 \%$. This was achieved by using a flow control system fed with $\mathrm{CO}_{2}$ and $\mathrm{N}_{2}$ inlets. Fig. 7 shows a preliminary evaluation of the sensor's response for the steep change. It is possible to observe the signal decreasing with the reduction of the gas concentration. From this result a recovery time of $50 \mathrm{~s}$ was estimated. Also, from the statistics of the acquired data, it is possible to state that the system is able to resolve a minimum $\mathrm{CO}_{2}$ change of $\pm 0.06 \%$, considering a minimum detectable signal of two times the standard deviation. It is worth to note that the system performance has a lot of room for improvement.

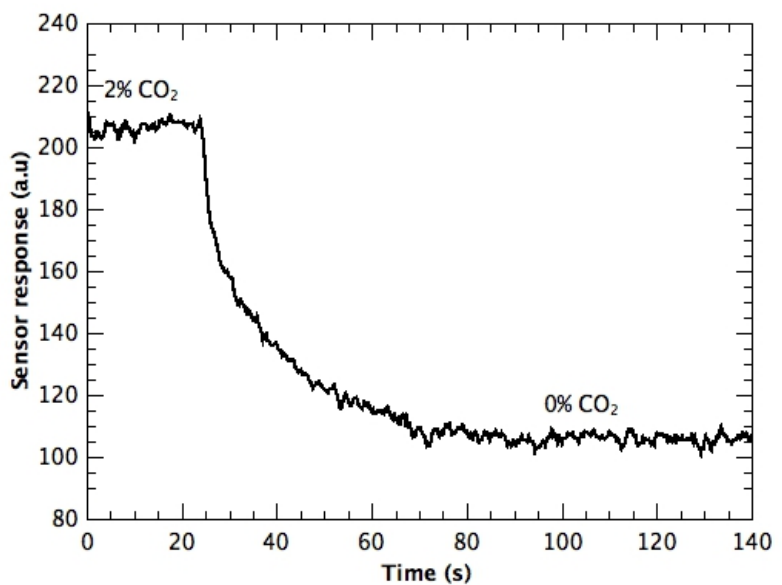

Fig. 7. Sensor response for a steep change of $2 \% \mathrm{CO}_{2}$, a resolution of approximately $0.06 \%$ can be estimated.

\section{B. Sensor Platform Performance}

The platform is able to adjust the power of the two lights in order to achieve a measurement reference point or zero measure. Later, by adjusting the switching frequency a desired signal gain can be manipulated, taking advantage of the active filter frequency response. These two features allow the optimized use of the 10 bit ADC, to improve the resolution of the measure. The platform is able to perform up to $1 \mathrm{kHz}$ data sampling, which allows digital filtering, if necessary, to remove unwanted noise. This will not affect the measurement response, as the sensitive colorimetric film has a much lower frequency sensitive response.

\section{CONCLUSION}

In this work an adjustable sensor platform was presented and explained how to achieve the adaptability and flexibility required when using colorimetric sensitive films. This was accomplished by a mixed analog and digital architecture, dividing the signal processing between them, resulting in a low cost and low power sensor platform. The combination of this platform with different sets of LEDs and chemically sensitive layers, provide a powerful platform for multiparemeter environmental monitoring systems.

The calibration process in this point is done manually but some degree of self-calibration is possible, taking advantage of the software adjustment and processing capabilities of the platform.

\section{ACKNOWLEDGMENT}

This work was supported by project Sensing «NORTE-070124-FEDER-000058», financed by the North Portugal Regional Operational Program (ON.2 - O Novo Norte), under the National Strategic Reference Framework (NSRF), by ERDF - European Regional Development Fund through the COMPETE Programme (operational programme for competitiveness) and by National Funds through the FCT Fundação para a Ciência e a Tecnologia (Portuguese Foundation for Science and Technology) within project «FCOMP-01-0124-FEDER-037281»

\section{REFERENCES}

[1] Jesus, C, P Caldas, O Frazao, J L Santos, PAS Jorge, and J M Baptista. 2009. "Simultaneous Measurement of Refractive Index and Temperature Using a Hybrid Fiber Bragg Grating/Long-Period Fiber Grating Configuration." Fiber and Integrated Optics 28 (6): 440-449.

[2] Wang, X, and O S Wolfbeis. 2012. "Fiber-Optic Chemical Sensors and Biosensors (2008-2012).” Anal. Chem., 2013, 85 (2), pp 487-508..

[3] McDonagh, Colette, Conor S Burke, and Brian D MacCraith. 2008. "Optical Chemical Sensors." Chemical Reviews 108 (2): 400-422.

[4] Gouveia, C. J., Markovics, A., Baptista, J. M., Kovacs, B., \& Jorge, P. A. S. (2011, May). Colorimetric and refractometric measurements of carbon dioxide. In International Conference on Applications of Optics and Photonics (pp. 80013C-80013C). International Society for Optics and Photonics.

[5] Gibson, Desmond, and Calum MacGregor. "A Novel Solid State NonDispersive Infrared CO2 Gas Sensor Compatible with Wireless and Portable Deployment." Sensors 13.6 (2013): 7079-7103.

[6] Pandey, Sudhir Kumar, and Ki-Hyun Kim. "The relative performance of NDIR-based sensors in the near real-time analysis of $\mathrm{CO} 2$ in air." Sensors 7.9 (2007): 1683-1696. 